\title{
Computing: The Shape of an Evolving Discipline
}

\author{
Lillian (Boots) Cassel [USA] (Co-Chair), Gordon Davies [UK] (Co-Chair), \\ Deepak Kumar [USA] (Rapporteur)
}

\section{Participants}

Ralf Denzer [Germany], Anneke Hacquebard [The Netherlands], Richard J. LeBlanc Jr. [USA], Luiz Ernesto Merkle [Brazil], Fred Mulder [The Netherlands], Zeljko Panian [Croatia], Ricardo Reis [Brazil], Eric Roberts [USA], Paolo Rocchi [Italy], Maarten van Veen [The Netherlands], Avelino Francisco Zorzo [Brazil]

\begin{abstract}
The task of defining a computing curriculum that has wide international application is a difficult one. Defining a common core among computing disciplines is complicated by the continuing growth of more diverse subdisciplines within computing and connections to a wider array of fields. The question whether we will be able to create a common understanding of excellence in our discipline is crucial for the quality of our education. In this report, we present an analysis of the current state of computing curriculum design and propose the development of a standard framework for looking at computing curricula, which could be used worldwide. For this purpose, a large-scale, multi-national effort will be necessary.
\end{abstract}




\section{INTRODUCTION}

The task of defining a computing curriculum that has wide international application is a difficult one. Despite the breadth that exists in recent international curriculum reports $[3,7,12]$, that diversity is easily surpassed by the enormous variation found in the programs of study that currently exist in various universities throughout the world. Defining a common core among computing disciplines like computer science, information systems, computer engineering, software engineering, and so forth is complicated by the continuing growth of more diverse sub-disciplines within computing and connections to a wider array of fields. The working group discussed these issues and arrived at the following two important problems that are of immediate concern:

1. Can we develop a process for reviewing and analysing new programs of study proposals and curriculum recommendations against some established standards?

2. Are there ways to compare curriculum recommendations and find commonalities and distinctions across national boundaries?

These questions pose urgent concerns in the wake of the delivery of several extensive curricular definition reports and others that will be delivered in the near future. In this report we outline the current state and trends in curriculum development as well as the challenges being faced, and we make recommendations for future directions that focus on these questions.

\section{CURRENT STATE}

In recent years, several important curricular design proposals have appeared in the literature, including the following:

- ICF-2000 (Informatics Curriculum Framework 2000) produced by IFIP by commission from UNESCO [12].

- CC2001 (Computing Curricula 2001) produced by ACM and IEEE-CS [7].

- Career Space (Curriculum Development Guidelines, New ICT Curricula for the $21^{\text {st }}$ century) developed by a consortium of 11 major ICT companies within the European Union (EU)[3].

The above list is not exhaustive. Other curricular documents exist and more are under active development. These curricula are quite diverse, reflecting the enormous variations in educational systems and philosophies throughout the world [11, 13]. A historical synthesis of curricular recommendations based on the diversity of the discipline of computing is 
presented in [9]. The underlying motivations for the design of various curricula range from knowledge-based curricula at one end of the spectrum to competency-based curricula at the other. While a number of the curriculum development efforts intend to be widely useful, all reflect the environments in which they are produced. Thus, CC2001 displays a focus on the United States educational system and ICF-2000 and Career Space are largely European $[3,11,13]$.

In addition to the curriculum definitions, there are also profile definitions that describe the variety of jobs available in the computing area [3, 6]. Several curricular evaluation procedures and benchmarks are also in existence, and more are in development (for example, see [4]). Like the curricular documents, these reports exhibit a wide range of international differences. Computing is a broad discipline that is increasingly difficult to define precisely. At the same time, the field has undergone a process of maturation over the past fifty years that allows curriculum designers to benefit from past experience.

\section{TRENDS}

The diversity of curricular recommendations clearly suggests that the discipline of computing continues to broaden its boundaries. Given the continuing rapid pace of advances in the discipline and the impact of computing on new and emerging disciplines and application contexts (for example, see [5] on a program on enviromatics), the domain of computing will continue to expand as its interaction with other disciplines increases.

National and cultural differences play an important role in the design of curricula. Despite these differences, we recognize that the curricular documents will play a defining role not only in the continents where they were designed, but also in several other international contexts. Already, there have been several instances of specific instantiations of curricula based on these recommendations in other countries [1, 10] with Computing Curriculum 2001 being used in Brazil and China.

In the EU, the Bologna Declaration (http://europa.eu.int/comm/ education/socrates/erasmus/bologna.pdf) is starting to have an effect. The declaration focuses on changes in the university educational systems in European countries to enable the mobility of students and the workforce. The goal is to create, in addition to the traditional degrees, a structure that substantially matches the Bachelor's and Master's degree programs in other countries. Further efforts to broaden the mobility of students and workers are needed in the light of increased communication and relocation. 
Despite the presence of specific curricular documents, there is a wide range of specific programs at various universities. An excessive number of degree program names within the domain of computing are found in the United Kingdom. While the problem is not as acute in other locations, the trend to more programs of study in computing is evident. Such innovation is essential to maintain the vitality of the discipline, but it raises the longstanding issue of the identity of the field.

Given the diversity of degree programs operating under the umbrella of computing, the issue of accreditation becomes more challenging. In the US, accreditation criteria have evolved significantly from rigid but measurable guidelines to more flexible and institution-centred evaluation. In the UK, a new quality assurance system based on the idea of benchmarks is being implemented $[4,8]$ and the British Computer Society is incorporating the benchmark for Computing in to their accreditation process. In both cases, accreditation is increasingly outcome-oriented, although some countries continue to use more quantitative standardized metrics. New accreditation guidelines have to be developed for universities in the EU because of changes being brought about by the Bologna Declaration. These developments are perceived as encouraging signs of increased cooperation, especially in the European context, such as the ECTS (European Credit Transfer System) approach.

\section{CHALlENGeS}

Perhaps the biggest challenge facing the community is to improve the understanding of various curricular recommendations and promote stronger cooperation among international communities. The goal of such an effort is to approach international applicability of various curricula. While some international communities have taken upon themselves to interpret and adapt already published curricula, we are also aware of resistance to adaptation in many other countries. We recognize that the existing curricular documents are a product of extensive research and substantial resources. Moreover, while most reflect a bias toward the educational systems of the countries in which they evolved, these curricula are excellent and comprehensive documents that can serve as a basis for defining curricula for other countries. We believe it is imperative to establish means for enabling comparison of curriculum recommendations and finding commonalities and distinctions across national boundaries. Can we develop a process for reviewing and analysing new programs and curriculum recommendations against some established standards? 
To answer this question, one of the first steps is to define the space that makes up the discipline of computing and characterise it in such a way as to accommodate its continuing evolution. Some of the salient elements of the characterizations will include:

- Body of Knowledge: The traditional starting point in curriculum design is to identify the knowledge areas required for that discipline. The body of knowledge must specify the critical topics along with specifications of pre-requisites and desired student outcomes.

- Foundational Material: Within each subdiscipline it is important to identify the foundational material that represents the core knowledge of that curriculum. This foundational material has long term relevance for the field and will remain important for many years.

- Application Context: While computing related content remains important within the narrow confines of building better computers, languages, operating systems and other artifacts of the computing environment, computing is also a significant part of many application domains. Partnerships among disciplines are essential to solve a variety of problems in every field of human endeavor. Curricula that address these needs must bring together essential elements from many disciplines as integrated parts of a single goal.

- Social Context: The social context of a curriculum is affected by cultural and economic conditions and can have a profound effect on the structure and approach of its presentation.

- Breadth and Depth: As the field continues to broaden, curriculum proposals must balance the breadth of the field with the inclusion of the required depth.

- Thematic Coherence: For every new program that is proposed, it is important to establish that the program makes thematic sense and that its goals are coherent and well established and not necessarily driven by market forces and transient trends

- Outcomes: Any curriculum proposal must be able to define the desired set of characteristics that its graduates must possess after completing the program. The specification of competencies of graduates must be sufficiently fine grained so that they can be combined in various ways to describe different types and levels of qualifications.

Each of these elements will have both quantitative and qualitative components. While it is important to identify measurable assessment strategies, one cannot ignore the qualitative aspects of curriculum design. It is also important to ensure that the definition of the space incorporates proper professional and social contexts rather than just the list of topics that traditionally comprise the body of knowledge. 
The accommodation and definition of new fields within computing should be facilitated to ensure sufficient transparency between curricula across national boundaries. We recognize that the dynamics of the discipline are different from other disciplines, especially as the discipline is starting to mature. We must attempt to take advantage of advances in pedagogy and also increase the level of interaction with appropriate stakeholders (that may include segments of industry as well as academic institutions in different countries). The process must also account for evaluation criteria that would facilitate explicit inclusion of issues surrounding diversity [2].

\section{FUTURE DIRECTIONS}

Defining ways to evaluate proposed curricula will require international cooperation. To define the space of valuable computing programs will require merging several existing bodies of knowledge. This would explicitly address the issues of competencies, the extensibility of the body of knowledge, the activities and experiences that would be a part of the curriculum, and the diversity of learning environments and delivery mechanisms that may be employed.

In an outcomes-based program evaluation, judgment must include an understanding of the result of an implementation the curriculum. Results can be expressed as profiles of graduates of the program that implements a particular curriculum recommendation. A profile may be related to a particular job description or to preparedness for further study in the field. The profile will consist of a set of competencies that are acquired through study and through experiences. Study is directly related to a body of knowledge and includes activities such as reading, discussion and practical work. The effect of study is demonstrated through examinations and other signs of accomplishment such as written reports or articles and presentations in various media. Experiences and activities are relevant to learning to use the body of knowledge in some context. These include laboratory activities, projects that require problem analysis, solution design and solution implementations. Relevant experiences can be achieved through class assignments, through internships and apprenticeships.

The process of curriculum evaluation should address different levels of outcomes based on student performance: threshold, modal, and top. A curriculum whose definition only addresses education for the average student will not include materials for developing the best students to their full potential. Thus, a fully developed curriculum will be expected to provide evidence of offering adequate opportunities for students at different levels. Students who perform at the topmost levels will receive appropriate 
challenges and opportunities to achieve competencies beyond those accessible by most students

There will be a need for several analysis tools to provide different ways of comparing programs to establish their strengths and weaknesses. These tools will include lists of topics from an extensible body of knowledge, competencies from which relevant profiles can be derived, experiences and activities that map topics to competencies, and ways of combining these elements and verifying that various conditions for consistency have been met. Such conditions will include the proper ordering of topic areas and experiences to assure that appropriate preparation precedes expected accomplishment; and evaluation of topic sets and experience to assure that sufficient breadth and depth are provided and lead to a meaningful profile. For example, the GRIP project is a comprehensive attempt at mapping job profiles with curricular descriptions [6]. Exploring such a process should be carried out at an international level to tap the expertise and experiences currently applied to different, but complementary projects. We expect this work to be carried out in a joint international project that combines efforts from many countries. While largely unifying in its goals, the project must be cognizant of national preferences, recognizing that curricula implementation will always take into account the local context and the experiences being provided to students using a wide variety of learning methodologies and environments.

\section{SUMMARY}

The answer to the question "Will we be able to create a common understanding of excellence in our discipline?" is crucial for the quality of our education. This can be achieved by developing a standard framework for looking at computing curricula, which could be used worldwide. For this purpose, a large-scale effort combining existing approaches is necessary.

In order to promote the health and benefits of the application of computing, it is essential to harness the energies and experiences of leaders in computing education around the world to produce a systematic method for comparing and merging curricular efforts and for assessing the potential contributions of proposed programs of study. 


\section{REFERENCES}

[1] Alvarez, J.: Innovations in Objectives, Content, Methodology and Grading in a First and Massive Computing Course, Keynote talk at of IFIP ICTEM 2002. Brazil, 2002.

[2] Blank, D., Kumar, D.: Patterns of Curriculum Design, Proceedings of IFIP ICTEM 2002, Brazil, 2002.

[3] Career Space: Curriculum Development Guidelines/New ICT Curricula for the $21^{\text {st }}$ Century: Designing Tomorrow's Education, available on the worldwide web at http://www.career-space.com and http://www.cedefop.gr, Office for Official Publications of the European Communities, CEDEFOP (European Centre for the Development of Vocational Training)/Career Space. 2001.

[4] Computing Benchmark Standard, published by the UK Quality Assurance Agency, Gloucester, England, 2000. www.qaa.ac.uk

[5] Denzer, R: A Computing Program for Scientists and Engineers: What is the core of Computing? Proceedings of IFIP ICTEM 2002, Brazil, 2002.

[6] GRIP (Generic Referential ICT Profiles), available on the worldwide web at http://www.grip-project.nl.

[7] IEEE-CS \& ACM: Computing Curricula 2001: Computer Science Volume, available on the worldwide web at: http://www.acm.org/sigcse/cc2001, IEEE-CS/ACM, 2001.

[8] McGettrick, A.: Benchmark Standards for Computing in the UK, Proceedings of IFIP ICTEM 2002 (IFIP Working Group 3.2 Conference on Informatics Curricula, Teaching Methods and Best Practice), Brazil, 2002.

[9] Merkle. L. E., Mercer, R. E.: Variations in Computing Science's Disciplinary Diversity: The case of curricula recommendations, Proceedings of IFIP ICTEM 2002, Brazil, 2002.

[10] Moraes, F. G., Zorzo, A. F., Calazans, N. L. V.: Deriving Different Computer Science Curricula from a Common Core of Disciplines, Proceedings of IFIP ICTEM 2002. Brazil, 2002.

[11] Mulder. F., Lemmen, K., van Veen, M.: Variety in Views of University Curriculum Schemes for Informatics/Computing/ICT, Proceedings of IFIP ICTEM 2002. Brazil, 2002.

[12] Mulder, F., van Weert, T. [eds], ICF-2000: Informatics Curriculum Framework for Higher Education. Paris, available on the worldwide web at http://www.ifip.or.at/ pdf/ICF2001.pdf UNESCO/IFIP, 2000.

[13] Roberts, E.: CC-2001 and the Challenge of Defining a Curriculum, Keynote talk at of IFIP ICTEM 2002. Brazil, 2002. 